\title{
Detecting Provenance Forgery and Packet Drop Attacks for Fire Detection System in Wireless Sensor Network
}

\author{
Daigo Menejis ${ }^{1}$, Sudarshan Kete ${ }^{2}$, Ranjeet Bhosale Patil ${ }^{3}$ \\ Student, Computer Engineering, Indira College of Engineering \&Management, Pune, India ${ }^{1,2,3}$
}

\begin{abstract}
Packet loss is possible in wireless sensor network. So that the intruders can be easily capture the data. Identifying the dropping packet and misbehaving activities are the most necessary measures for secure transmission in it. Without a certificate a node cannot participate in the transmission. In our project, we present Sensors which can senses the Data From environment and data will send by using Arduino to other computer.In that system we provide an efficient and secure approach for transmitting provenance information about sensor data. Our approach uses packet Bloom filters that are encoded as sensor data travels via intermediate sensor nodes, and are decoded and verified at the Receiver. Thus Information Gain by Receiver will Trace out the data Path through which it comes .We can used this system in Fire detection Application .In this Application Whatever the fire detection Sensors For EX., Flame Sensors, Smoke Sensors senses the Information from Environment this Information need to transfer at Authorized persons or else want to send at Server. In between this transmission attacker can attack any of packets of Data or Data may loss due to collusion occurred. In this situation we proposed the this model to trace the Attackers and find out the data path from where the data is going .
\end{abstract}

Keywords: Provenance Model, Security, Sensor Networks, Bloom Filter Packet generation, Fault location.

\section{INTRODUCTION}

In this project, we tend to present AN economical and secure approach for transmission origin data concerning device information. Our provenance approach uses light-weight in-packet Bloom filters that are encoded as device information travels through intermediate device nodes, and are decoded and verified at the bottom station. Our origin technique is additionally able to defend against malicious attacks like packet dropping and permits one to sight the accountable node for packet drops. Intrinsically it makes attainable to change the transmission route to avoid nodes that would be compromised or defective. Additionally, we tend to expand the system to scientific discipline tracing i.e. That forward the information to base station once malicious nodes compromise the intermediate node. Our technique is meant to make a trustworthy surroundings for device nodes wherever solely trusty information is processed. We tend to assess the planned system each analytically and through an experiment, and also the outcomes demonstrate the adequacy and potency of the light-weight secure origin theme detection packet forgery los attacks yet as scientific discipline tracing.

\section{MOTIVATION}

In a multi-hop detector network by victimisation knowledge rootage theme the baccalaureate will trace the supply and forwarding path of a private data packet. for every packet rootage should be recorded however there's a very important challenge arises as a result of the significant storage, energy and information measure conditions of detector nodes. So, it's necessary to supply a light-weight rootage theme with low overhead.As against existing analysis that employs separate transmission channels for information and rootage, we tend to solely need one channel for each. moreover, ancient rootage security solutions use intensively cryptography and digital signatures, and that they use append-based information structures to store rootage, resulting in prohibitory prices. In distinction, we tend to use solely quick Message Authentication Code (MAC) schemes and Bloom filters (BF), that area unit fixed-size information structures that succinctly represent rootage. Bloom filters build economical usage of information measure, and that they yield low error rates in observe.

\section{III.LITERATURE REVIEW}

1. Trustworthiness Assessment of Knowledge on the Semantic Sensor Web by Provenance Integration:

Knowledge depicted on the linguistics sensing element net originates from totally different datasets that square measure usually a set or aggregation of alternative sources. The point is dynamic, open and distributed, that the datasets square measure of varied quality and completeness. Customers got to be given grade of trait of this information to see its 
connexion and utility. Interpretation of rootage (detailed data regarding the origin of knowledge is important so as to analyse however knowledge came into existence and live its trait. But there square measure challenges in deciphering the rootage in a very uniform method, as a result of totally different information suppliers use different processes to govern the information and different annotation techniques to produce data. Though there square measure ways for retrieving rootage, data customers square measure left with the responsibility of assessing the trait of discovered data passionate about however they see it fitting their application. This paper proposes a meta-knowledge metaphysics to align the ideas and properties of existing rootage schemas and ontologies. The meta-provenance metaphysics permits common interpretation of various provenances, and thus their integration.

\section{Provenance-Based Information Trustworthiness Evaluation in Multi-Hop Networks:}

In this paper, we tend to gift a trust model to guage the trustiness of data similarly because the information business enterprise nodes supported the data beginning. we tend to think about 2 factors in evaluating the provenance-based data trust: Path Similarity and data Similarity. In multihop networks, data will flow through multiple hops from multiple ways. we tend to model the similarity between completely different ways that deliver data regarding constant event and therefore the similarity between 2 data things regarding constant event that ar delivered through different ways. each path and data similarity factors are thought-about in decisive the trust of the data. This data trust is so used as a feedback issue to adaptively alter trust of the nodes within the network. elaborated analysis of the projected approach is conferred together with simulation results for validation.

\section{Chimera: a virtual data system for representing, querying, and automating data derivation :}

A lot of scientific knowledge isn't obtained from measurements however rather derived from alternative knowledge by the appliance of procedure procedures. We have a tendency to hypothecate that express illustration of those procedures will change documentation of information place of origin, discovery of obtainable strategies, and on-demand knowledge generation (so-called "virtual data"). To explore this concept, we've developed the Chimera virtual information system, which mixes a virtual knowledge catalog for representing knowledge derivation procedures and derived knowledge, with a virtual knowledge language interpreter that interprets user requests into knowledge definition and question operations on the info. We have a tendency to couple the Chimera system with distributed "data grid" services to change on-demand execution of computation schedules made from info queries. We've applied this method to 2 challenge issues, the reconstruction of simulated collision event knowledge from a high-energy physics experiment, and looking out digital sky survey knowledge for galactic clusters, with promising results.

\section{Towards Provenance Aware Design of Service Compositions: A Methodology for Analysing the Provenance Awareness in Service Designs:}

And analysing non-functional properties (nfps) is important for driving field choices and validatory composite service styles. Solely wherever nfps are fixed will we elect between services with similar practicality that will higher satisfy our non-functional necessities. Meanwhile, incorporating rootage practicality into service-oriented systems' style is turning into crucial for users, permitting them to question the generation strategies and origins of the info the system outputs. This would like is especially evident in compositions of services, wherever audits of individual services don't give a connected image of the composition's process history. Creating rootage awareness (ability to answer rootage queries) an exact NFP in composite service specifications would change composite service designers to analyse whether or not they meet provenance-related necessities. During this paper, we tend to discuss a framework for coming up with and analysing rootage awareness for service compositions. We tend to envision this as a basis for analysing the impact of rootage on different nfps like performance and storage.

\section{Provenance-Aware Sensor Data Storage:}

Sensor network information has each historical and realtime worth. Creating historical device information helpful, specially, needs storage, naming, and categorization. Device information presents new challenges in these areas. Such information is location-specific however additionally distributed; it's collected during a explicit physical location and should be most helpful there, however it's extra worth once combined with alternative device information collections during a larger distributed system. Thus, transcription location-sensitive peer-to-peer storage is one challenge. Device information sets don't have obvious names, therefore naming them during a globally helpful fashion is another challenge. The last challenge arises from the requirement to index these device information sets to create them searchable. The key to device information identity is beginning, the complete history or lineage of the information. We tend to show however beginning addresses the naming and categorization problems so gift a pursuit agenda for constructing distributed, indexed repositories of device information.

\section{IV.PROPOSED SYSTEM}

In this project, we have a tendency to present associate degree economical and secure approach for sending origin info concerning sensing element knowledge. Our provenance approach uses light-weight in-packet Bloom filters that are 
encoded as sensing element knowledge travels through intermediate sensing element nodes, and are decoded and verified at the bottom station. Our provenance technique is additionally ready to defend against malicious attacks like packet dropping and permits one to find the accountable node for packet drops. intrinsically it makes doable to switch the transmission route to avoid nodes that might be compromised or malfunctioning . additionally, we have a tendency to expand the system to informatics tracing i.e. that forward the info to base station once malicious nodes compromise the intermediate node. Our technique is meant to make a trustworthy atmosphere for sensing element nodes wherever onlytrusted knowledge is processed. we have a tendency to assess the planned system each analytically and by experimentation, and therefore the outcomes demonstrate the adequacy and potency of the light-weight secure provenance theme in detection packet forgery and los attacks in addition as informatics tracing.

In our Project We have Implemented To Find The Attacks on Data Sent Over Source node to the Receiver Node .Fig.1 Shows the View of Attacker attacks the Data Between the Source and destination.

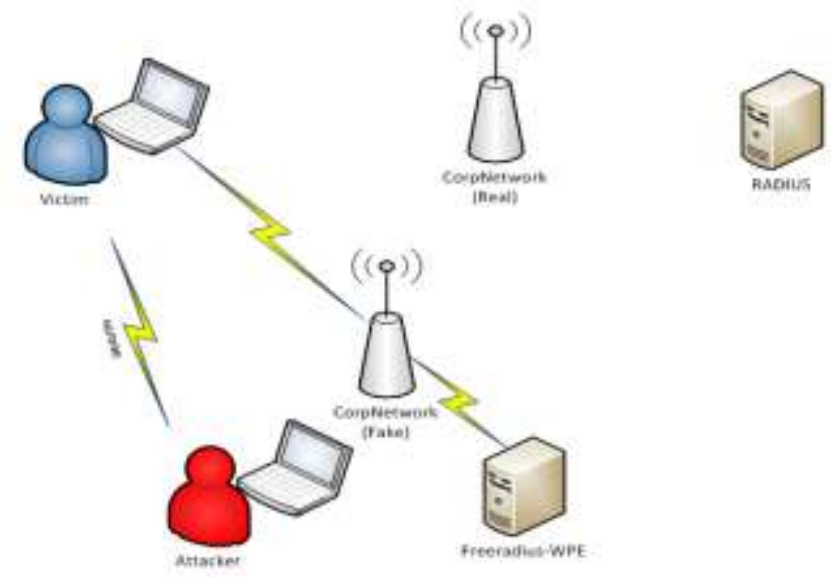

Fig.1Attacker attacks the Data Between Nodes

FEATURES:

$>$ Packet generation: It generates the packet set from totally different heat sensing element.

$>$ Bloom Filter: It filter Probabilistic set of packets. it's at receiver aspect.

$>$ Fault location: It detects the attacks done by that go-between.

$>$ Provenance encoding :Encoding packet for security.

> Provenance decoding: decoding sensing element information packet at the base station.

Following Fig.2 Shows The Architecture of Our Proposed System.

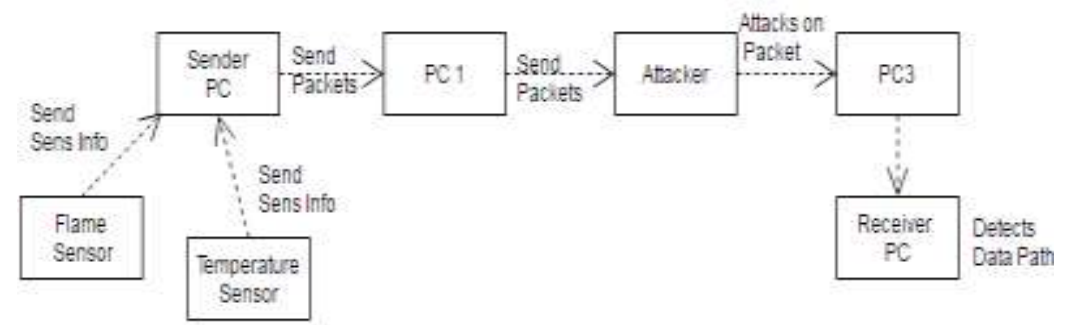

Fig.2 Architecture view of Proposed System

\section{IMPLEMENTATION}

The Bloom Filter (BF):

The BF is a space-efficient arrangement for probabilistic representation of a collection of things $\mathrm{S}=$ using an array of $\mathrm{m}$ bits with $\mathrm{k}$ freelance hash functions $\mathrm{h} 1, \mathrm{~h} 2, \ldots, \mathrm{hk}$. The output of every hash operate hi maps associate item s uniformly to the vary $[0, \mathrm{~m}-1]$, i.e., associate index during a m-bit array. The BF may be diagrammatical as . ab initio all $\mathrm{m}$ bits are set to zero. To insert part $\mathrm{s} \in \mathrm{S}$ into a $\mathrm{BF}, \mathrm{s}$ is hashed with all the $\mathrm{k}$ hash functions manufacturing the values his $(1 \leq$ $\mathrm{i} \leq \mathrm{k})$. The bits adore these values are then set to one within the bit array. Figure two illustrates associate example of BF insertion. To query the membership of associate item thus inside $\mathrm{S}$, the bits at indices $\mathrm{hi}\left(\mathrm{s}_{\_}\right)(1 \leq \mathrm{i} \leq \mathrm{k})$ are checked. If any of them is zero, then definitely thus $\_\in \mathrm{S}$. Otherwise, if all of the bits are set to one, thus $\in \mathrm{S}$ with high likelihood. There exists a clear stage of error that arises thanks to hashing collision that creates the weather in $\mathrm{S}$ conjointly inflicting indices hi(s_) being set to one even though thus _ $\in \mathrm{S}$. 
This is often referred to as a false positive. Note that, there's no false negative within the BF membership verification

\section{SECURE PROVENANCE ENCODING:}

We propose a distributed mechanism to encrypt provenance at the nodes and a centralized formula to decipher it at the BS. The technical core of our proposal is that the notion of in-packet Bloom filter (iBF). every packet consists of a novel sequence range, data value, associated an $\mathrm{BBF}$ that holds the place of origin. we tend to emphasize that our focus is on firmly sending cradle to the BS. In associate aggregation infrastructure, securing the info values is additionally a very important side, however that has been already addressed in previous work. Our secure root technique are often employed in conjunction with such work to get a whole resolution that gives security for information, provenance and data-provenance binding.

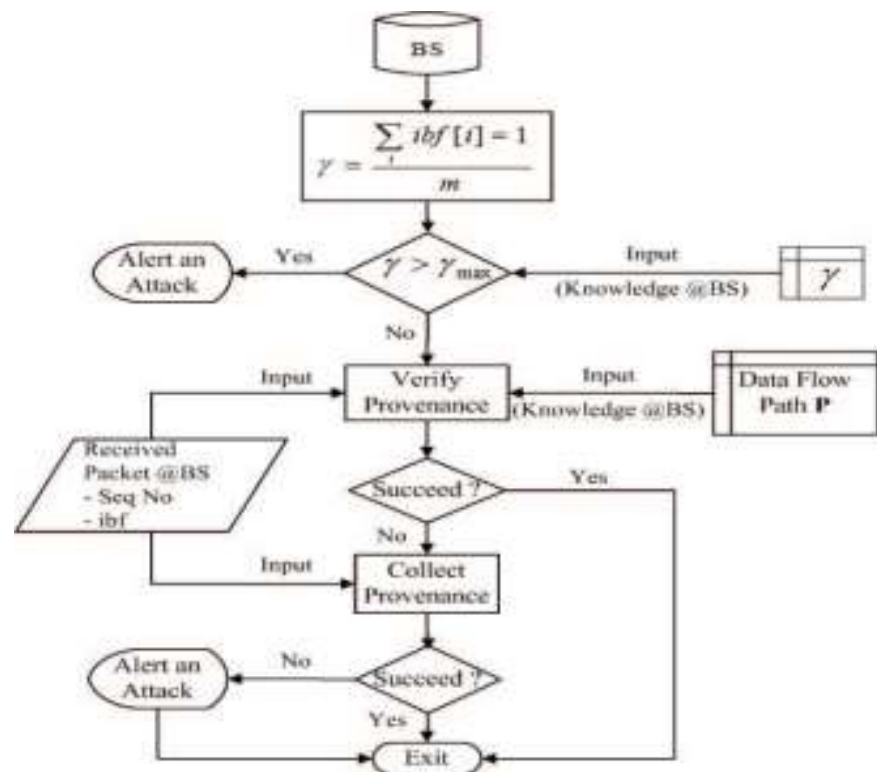

Fig.3 Flow of System

\section{AES Encryption:}

AES may be a centrosymmetric coding formula.The formula was developed by two Belgian decipherer Joan Daemen and Vincent Rijmen.AES was designed to be economical in each hardware and computer code, and supports a block length of 128 bits and key lengths of 128, 192, and 256 bits. AES coding is employed by U.S. for securing sensitive however unclassified material, therefore we are able to say it's enough secure. When you wish to write a confidential text into a decryptable format, for instance once you got to send sensitive knowledge in e-mail. The coding of the encrypted text it's doable given that you recognize the proper arcanum. If you would like to write a text place it within the white textarea on top of, set the key of the coding then push the write button.The results of the coding can seem in base64 encoded to stop character secret writing issues. If you would like to rewrite a text make certain it's in base64 encoded and is encrypted with AES algorithm! Put the encrypted text within the white textarea, set the key and push the rewrite button.

\section{VI.RESULT ANALYSIS}

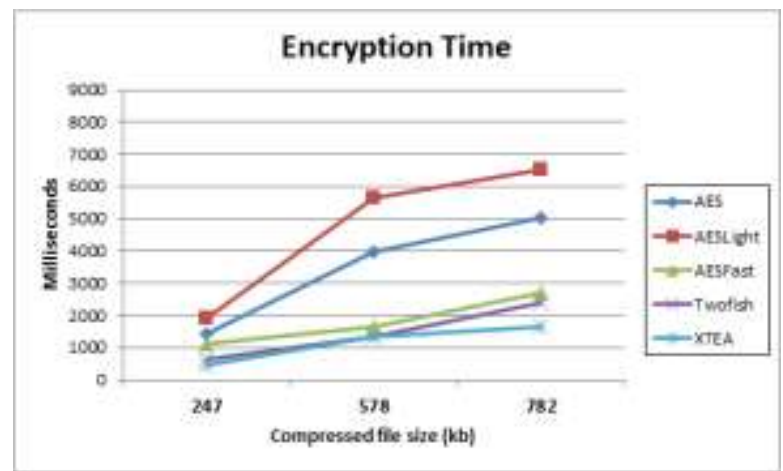

Fig.4 AES Encryption Analysis With other 

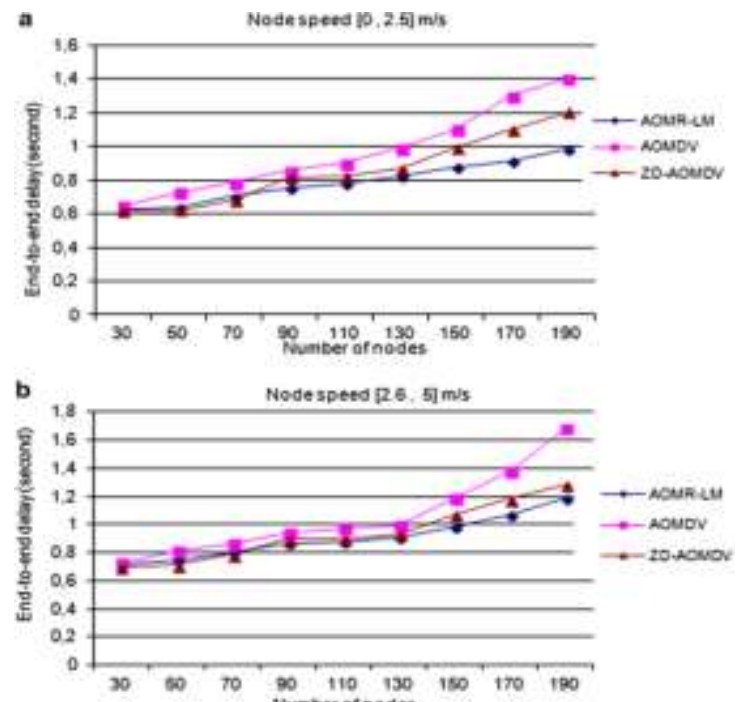

Fig. 5 Transmission speed of Packets through mediator

\section{CONCLUSION}

In this we tend to addressed the matter of firmly transmittal fire connected sensing element data in wireless device networks, and planned a provenance coding and decoding theme supported Bloom filters. The theme ensures confidentiality, integrity and freshness of sensing element information. We tend to embody packet sequence data that supports detection of packet loss attacks. This captures origin for network packets within the variety of per packet tags that store a history of all nodes and processes that manipulated the packet. However, the theme assumes a trusty surroundings that isn't realistic in sensing element networks. It describes the history and derivations of network state that result from the execution of a distributed protocol.

\section{ACKNOWLEDGMENT}

The heading of the Acknowledgment section and also the References section should not be numbered. It offers us nice pleasure in presenting the preliminary project report on 'Detecting provenance Forgery And Packet Drop Attacks For fireplace Detection System In Wireless sensor Network. I might wish to take this chance to impart my internal guide for giving me all the assistance and steering I required. I'm very grateful to them for his or her kind support. Their valuable suggestions were very useful. I am additionally grateful to Head of COMPUTER ENGINEERING Department ,ICOE for his indispensable support, suggestions. In the finish our special due to different Person Name for providing numerous resources like laboratory with all required software system platforms, continuous internet association, for Our Project.

\section{REFERENCES}

[1] Salmin Sultana, Gabriel Ghinita, "A Lightweight Secure Scheme for Detecting Provenance Forgery and Packet Drop Attacks in Wireless Sensor Networks",Ieee transaction on dependable and secure computing Vol:6,No:1,Jan 2015.

[2] H. Lim, Y. Moon, and E. Bertino, "Provenance-based trustworthiness assessment in sensor networks," in Proc. of Data Management for Sensor Networks, 2010, pp. 2-7.

[3] I. Foster, J. Vockler, M. Wilde, and Y. Zhao, "Chimera: A virtual data system for representing, querying, and automating data derivation," in Proc. of the Conf. on Scientific and Statistical Database Management, 2002, pp. 37-46.

[4] K. Muniswamy-Reddy, D. Holland, U. Braun, and M. Seltzer, "Provenance-aware storage systems," in Proc. of the USENIX Annual Technical Conf., 2006, pp. 4-4.

[5] Y. Simmhan, B. Plale, and D. Gannon, "A survey of data provenance in e-science," SIGMOD Record, vol. 34, pp. 31-36, 2005

[6] R. Hasan, R. Sion, and M. Winslett, "The case of the fake picasso: Preventing history forgery with secure provenance," in Proc. Of FAST, 2009, pp. 1-14.

[7] S. Madden, J. Franklin, J. Hellerstein, and W. Hong, "TAG: a tiny aggregation service for ad-hoc sensor networks," SIGOPS Operating Systems Review, no. SI, Dec. 2002. 